\title{
Editorial
}

\section{Impacto del cribado del cáncer de próstata sobre la mortalidad}

\author{
Juan Morote Robles
}

Servicio de Urología y Trasplante Renal. Hospital Vall d'Hebron. Universidad Autonóma de Barcelona. Barcelona.

$\mathrm{R}^{\mathrm{e}}$ ecientemente, han sido publicados en la revista NEJM los resultados de dos importantes estudios, uno realizado en Estados Unidos ${ }^{1}$ y el otro en Europa ${ }^{2}$ cuyo objetivo principal fue analizar el impacto del cribado del cáncer de próstata sobre la mortalidad. Los resultados de ambos estudios son contradictorios y sin embargo; su trascendencia es tan relevante que, probablemente sea la única oportunidad que la comunidad científica haya tenido para demostrar la utilidad del cribado del cáncer de próstata.

El efecto de cualquier cribado a corto plazo es el incremento en la incidencia de la patología en cuestión y esto ya fue observado a los pocos años de la utilización generalizada del PSA. A medio plazo, el incremento en la supervivencia es otro efecto del cribado, que también ha sido confirmado. Sin embargo, el incremento en la supervivencia puede ser simplemente la expresión del sesgo producido por el momento en el que se produce el diagnóstico. Por ello, la disminución de la mortalidad es el único efecto a largo plazo que finalmente puede justificar un cribado. En mi opinión el reto que tiene el cribado del cáncer de próstata va todavía más allá, ya que ésta es una neoplasia que se presenta generalmente en hombres de edad avanzada y además tiene una lenta historia natural. Un dato que nos pone sobre aviso acerca del impacto del cáncer de próstata sobre la mortalidad es el número de años de vida potencialmente perdidos de que es causa y podemos observar que es muy inferior al que otras neoplasias producen. En otras palabras, podría producirse la paradoja de que reduciendo la mortalidad por cáncer de próstata no se redujera la mortalidad global, o sea, el cribado podría estar solamente produciendo un cambio en la causa de muerte en la población masculina.

El objetivo de esta editorial es presentar un análisis inteligible de lo que ambos estudios aportan y extraer algunas conclusiones prácticas.

El primer artículo reporta los resultados iniciales del PLCO sobre mortalidad por cáncer de próstata y llama poderosamente la atención la simple lectura del resumen. Entre 1993 y 2001 se randomizaron 76,693 hombres a realizar, en el grupo de cribado $(38,343)$, una determinación anual de PSA durante 6 años y un TR durante 4 años, y en el grupo control $(38,350)$, el cuidado habitual que curiosamente incluía en ciertas áreas el cribado del cáncer de próstata, recomendado por algunas organizaciones. En el apartado de resultados se especifica que el cumplimiento en el grupo de cribado fue alrededor del $85 \%$ mientras que en el grupo control la tasa de cribado con PSA fue del $40 \%$ durante el primer año y llegó al 52\% en el sexto año. En relación al TR, los niveles de contaminación fueron del $41 \%$ y $46 \%$ respectivamente. Tras 7 años de seguimiento, se detectaron 2.820 cánceres en el grupo de cribado y 2.322 en el grupo control, siendo la razón entre ambos 1,22 (95\% IC 1,16-1,29). Durante el mismo periodo se confirmaron 52 muertes por cáncer de próstata en el grupo cribado y 44 en el grupo control, siendo la razón entre ambas 1,13 (95\% IC 0,751,70). Finalmente, se comenta el cumplimiento del seguimiento había sido del $67 \%$ a los 10 años y que los resultados fueron similares a los reportados a 7 años. El resumen concluye que la tasa de mortalidad fue baja y que no se diferenció entre ambos grupos.

Al sumergirnos entre los datos que se reportan este estudio se confirma que la primera causa de muerte no específica en pacientes con cáncer de próstata es la patología cardiocirculatoria, que alcanza el 41,7\% (1648 de 3953 muertes) en el grupo de cribado y el 41,3\% (1675 de 4058 muertes) en el grupo control. Al comparar la mortalidad específica con la mortalidad no específica, nuestra reflexión es que el impacto de la mortalidad por cáncer de próstata sobre la mortalidad global en varones los varones estudiados fue mínimo. La mortalidad por cáncer de próstata representó solamente el 1,3\% en el grupo de cribado y el 1,1 en el grupo control. La reflexión sobre estos datos nos lleva a pensar que, al margen de la contaminación, el impacto de la mortalidad por cáncer de próstata sobre la mortalidad global cuando el diagnóstico se hace precozmente es muy bajo. Quizá, no debería plantearse el cribado del cáncer de próstata en un segmento de edad tan avanzado. 
El segundo aspecto del estudio americano que nos gustaría comentar es el grado de contaminación. En el apartado de material y métodos, no se especifica qué tasa de contaminación era la esperada, ni su posible impacto. No obstante, en la discusión se comenta que el nivel de contaminación en el grupo control fue suficiente como para diluir cualquier modesto efecto del cribado. A continuación se comenta que en el diseño original del estudio se estimó una contaminación del $20 \%$ y que el nivel en el reporte final fue similar al 38\% detectado en la revisión del protocolo realizada en 1998. En nuestra opinión, una tasa de detección en el grupo de cribado solamente incrementada en un $22 \%$ y la verificación de una distribución prácticamente igual respecto al estadio clínico en ambos grupos, claramente justifica que se están comparando dos poblaciones muy similares y por ello se confirmaría el impacto de la elevada contaminación de este estudio que llegó al 52\% en el sexto año de seguimiento. Este fenómeno debería invalidar los resultados de estudio, siendo ésta la verdadera conclusión y como consecuencia la imposibilidad de obtener respuesta al objetivo primario de este estudio

El segundo artículo recoge los datos del ERSPC, reportados como consecuencia de haber sido detectadas diferencias estadísticamente significativas por un comité independiente. En este estudio se randomizaron 162,387 hombres con una edad comprendida entre 55 y 69 años, 72,890 en el grupo de cribado y 89,353 en el grupo control, reclutados en 7 países europeos con unos criterios para la realización de biopsia no uniformes, y un seguimiento medio de 9 años. En el diseño del estudio se estimó un poder del 86\% para detectar una diferencia significativa del $25 \%$ o mayor en mortalidad por cáncer de próstata, asumiendo un nivel de cumplimiento del $82 \%$ en el grupo de cribado y un $20 \%$ de contaminación. La incidencia de cáncer de próstata detectada en el grupo de cribado fue $8,2 \%$ y 4,8 en el grupo control. La tasa de mortalidad específica por 1.000 hombres relativa del grupo de cribado respecto al grupo control fue 0,80 (95\% IC 0,65-0,98; $\mathrm{p}=0,04)$. Esto significa que 1.410 hombres deberían someterse a cribado y adicionalmente, 48 pacientes tratados para evitar una muerte por cáncer de próstata. Finalmente, la conclusión es que el cribado basado en PSA reduce la mortalidad específica un 20\%, pero asociado a una elevada tasa de sobrediagnóstico.

Durante el periodo de seguimiento, se confirmaron 214 muertes por cáncer de próstata en el grupo de cribado y 326 en el grupo control. Las curvas de mortalidad inician una separación creciente a partir de los 7 años de seguimiento. Esta evolución de la mortalidad es, en nuestra opinión, coherente con el efecto del cribado sobre una neoplasia con una lenta historia natural, sien- do esperable que las diferencias vayan aumentando con el paso del tiempo. Los datos reportados en los apéndices también muestran una distribución del estadio clínico que pone de manifiesto una migración del estadio a fases más iniciales en el grupo cribado. Finalmente, el análisis del efecto de la edad sobre el cribado pone de manifiesto que la reducción de la mortalidad fue significativa en todos los segmentos de edad, entre 55 y 69. Sin embargo, el efecto no observó entre 70 y 74 años. Entre 50 y 54 tampoco se detectó diferencia, aunque el número de muertes en este grupo fueron solamente 6 y 4 respectivamente.

Finalmente se concluye en este artículo que el sobrediagnóstico y sobretratamiento son probablemente los efectos adversos más importantes del cribado del cáncer de próstata, siendo estos efectos mucho más comunes que en los cribados del cáncer de mama, colorrectal o cervical.

Mi reflexión inicial al leer ambas publicaciones es que el estudio americano está comparando dos poblaciones con una elevada tasa de cribado y como consecuencia no es útil para analizar el objetivo primario de estudio. En relación al estudio europeo, se pueden objetar muchas razones para cuestionar la validez de sus resultados. Sin embargo, respecto al objetivo principal del estudio creo puede concluirse que el cribado del cáncer de próstata reduce la mortalidad específica cuando se realiza antes de los 70 años. No obstante, no se debe ser optimista en cuando a la bondad absoluta del cribado ya que el sobrediagnóstico es una consecuencia importante. En mi opinión la utilidad del cribado del cáncer de próstata pasará en el futuro por mejorar la especificidad de los métodos de diagnóstico y la eficacia para detectar los cánceres clínicamente relevantes además de focalizarlo sobre una población más joven de la actualmente considerada. $\mathrm{El}$ estudio europeo provee la primera evidencia científica de alto nivel a favor del cribado del cáncer de próstata, aunque deja un camino abierto para mejorar algunos aspectos de gran trascendencia.

\section{REFERENCIAS}

1. Andriole GL, Grubb III LB, Buys SS, ChiaD, Church TR, Fouad NN et al for the PLCO Project Team. Mortality Results from a Randomized ProstateCancer Cribado Trial. N Engl J Med 2009;360(13):1310-1319.

2. Schröder FH, Hugosson J, Roobol MJ, Tammela TL, Ciatto S, Nelen V et al for the ERSPC Investigatorss. Cribado and Prostate-Cancer Mortality in a Randomized European Study. N Engl J Med 2009;360(13):1320-1328.

Correspondencia autor: Dr. Juan Morote Robles

Servicio de Urología y Trasplante Renal. Hospital Vall D’Hebrón. Universidad Autónoma de Barcelona

Po Vall d’Hebrón, 119-129 - 08035 Barcelona. Tel.: 932746000

E-mail autor: jmorote@vhebron.net

Información artículo: Editorial

Trabajo recibido: mayo 2009 